\title{
Modelling for The COVID-19 with The Contacting Distance
}

\section{Zhihui Ma ( $\nabla$ mazhh@lzu.edu.cn )}

Lanzhou University

\section{Shufan Wang}

Northwest Minzu University

Xuanru Lin

Lanzhou University

\section{Xiaohua Li}

Lanzhou University

Xiaotao Han

Northwest Minzu University

Haoyang Wang

McMaster University

Hua Liu

Northwest Minzu University

\section{Research Article}

Keywords: COVID-19, Contacting distance, Immigration rate, Sensitivity analysis, Numerical test, Control method.

Posted Date: July 19th, 2021

DOI: https://doi.org/10.21203/rs.3.rs-329034/v2

License: (c) (i) This work is licensed under a Creative Commons Attribution 4.0 International License.

Read Full License 


\title{
Modelling for the COVID-19 with the contacting distance
}

\author{
Zhihui Ma ${ }^{1 *}$, Shufan Wang ${ }^{2}$, Xuanru Lin ${ }^{1}$, Xiaohua $\mathrm{Li}^{1}$, Xiaotao $\mathrm{Han}^{2}$, \\ Haoyang Wang ${ }^{3}$, Hua Liu $^{2}$ \\ 1. School of Mathematics and Statistics, Lanzhou University, \\ Lanzhou, Gansu 730000, People's Republic of China \\ 2. School of Mathematics and Computer Science, Northwest Minzu University, \\ Lanzhou, Gansu 730000, People's Republic of China \\ 3. Faculty of Science, McMaster University, Hamilton, Ontario, L8S4L8, Canada
}

\begin{abstract}
The COVID-19, which belongs to the family of Coronaviridae and is large-scale outbreak in the whole world, is a public health emergency for human beings and brings some very harmful consequences in social and economic fields. In order to modelling the COVID-19 and develop the efficient control method corresponding to the contacting distance, this paper proposes an SEIR-type epidemic model with the contacting distance between the healthy individuals and the asymptomatic or symptomatic infected individuals, and the immigration rate of the healthy individuals, since the contacting distance and the immigration rate are two critical factors which determine the transmission of the COVID-19. Firstly, the threshold contacting distance and the threshold immigration rate are obtained by analyze the dynamical behaviors of the proposed SEIR-type epidemic model in order to control the COVID-19. The effect of the contacting distance and the immigration rate on the control of the COVID-19 are revealed based on ecological and epidemiological issues. The results show that the COVID19 will be controlled while the contacting distance between the healthy individuals and the symptomatic infected individuals is larger than the threshold value $\tilde{d}^{*}$ and the immigration rate is smaller than the threshold value $\tilde{A}^{*}$. Secondly, the sensitivity analysis is conducted and the results show that the contacting distance and the immigration rate play an important

*Corresponding author: mazhh@lzu.edu.cn
\end{abstract}


2 Zhihui Ma, Shufan Wang, Xuanru Lin, Xiaohua Li, Xiaotao Han, Haoyang Wang, Hua Liu

role in controlling the COVID-19. Finally, the numerical test for Wuhan city are conducted and the conclusions show that the extinct lag decreases as the the contacting distance increase or the immigration rate decrease. Our study could give some reasonable suggestions for the health officials and the public.

Keywords: COVID-19, Contacting distance, Immigration rate, Sensitivity analysis, Numerical test, Control method.

\section{Background}

Coronaviruses are the enveloped, single-stranded, positive-sense RNA viruses and belong to the family of Coronaviridae (Chen et al., 2020; Tang et al., 2020). They could induce wildly respiratory infections, even though are accompanied by the relatively low mortality. Since their discovery and first characterization by the United Kingdom in 1965 (Kahn and McIntosh, 2005), two large-scale outbreaks have occurred and induced the public health events. For examples, the Severe Acute Respiratory Syndrome (SARS) in 2003 in mainland China (Hui and Zumla, 2019), and the Middle East Respiratory Syndrome (MERS) in 2012 in Saudi Arabia (Wit et al., 2016; Willman et al., 2019; Killerby et al., 2020). These outbreaks have resulted in more than 8, 000 and 2, 200 confirmed SARS and MERS cases, respectively [Kwok et al., 2019; Chen et al., 2020].

At the end of 2019, a third bigger outbreak occurred in the whole world. On December $29^{t h}$ 2019, four cases were reported by the Wuhan Municipal Health Commission (WMHC) and all confirmed cases are seemingly linked to the Huanan (Southern China) Seafood Wholesale Market (HSWM) (Cheng et al., 2020; Li et al., 2020; Rothe et al., 2020; Zhao et al., 2020). These reported cases were firstly identified as the novel coronavirus-infected pneumonia, and then the World Health Organization (WHO) named this infectious disease as the coronavirus disease 2019, being simplified as the COVID-19, on February 11 ${ }^{\text {th }}, 2020$ (Li et al., 2020; WHO Situation Report-22, 2020). On January $20^{t h}, 2020$, the Chinese government revised the law provisions about COVID-19 and defined it as class B agent. Public health officials announced a further revision to classify it as class A agent. Some non-pharmaceutical interventions, including the intensive contact tracing followed by quarantine of individuals potentially exposed to the infected peoples, the isolation of the symptomatic infected individuals and so all, were subsequently adopted, and then gave some positive effect on control of the COVID-19.

The COVID-19 has already caused a huge economic loss due to national lock-downs, travel restrictions, and global distractions of trade and manufacturing chains, and a large number of deaths due to the shortage of medical resources. Therefore, the COVID-19 is a seriously public 
health emergency for the whole world and brings some very harmful consequences in social and economic fields (Kwok et al., 2019; Cheng et al., 2020; Li et al., 2020; Rothe et al., 2020; Chen et al., 2020; WHO Situation Report-112, 2020; Zhao et al., 2020). In order to prevent and control the COVID-19 effectively, lots of researchers have conducted to study the COVID-19 based on medicine, epidemiology, biology and mathematics (Cheng et al., 2020; Li et al., 2020; Rothe et al., 2020; Tang er al., 2020; Zhao et al., 2020). Li et al. (Li et al., 2020) collected the confirmed information on demographic characteristics, exposure history, and illness timelines of laboratoryconfirmed cases that had been reported on January $22^{t h}$, 2020. They described characteristics of the cases and estimated the key epidemiologic time-delay distributions (the 95th percentile of the distribution at 12.5 days), the epidemic doubling time ( $D T=7.4$ days $)$ and the basic reproductive number $\left(R_{0}=2.2(95 \% \mathrm{CI}, 1.4-3.9)\right)$. Fortunately, in the early period, this research have successfully characterized and predicted some critical properties of the COVID-19. However, as the development of the COVID-19 and the adoptions of some effective prevention and control measures, the COVID-19 should be re-estimated. Tang et al. (Tang et al., 2020) presented a general SEIR-type epidemiological model and estimated the basic reproduction number by means of mathematical modeling. Their results showed that the control reproduction number might be as high as 6.47 (95\% CI, 5.71-7.23). As recognized by the World Health Organization (WHO), the mathematical models, which are employed to describe the COVID-19, play an important role for the disease prevention and control, and the policy formation.

The published researches have shown that the main transmission route of the COVID-19, including all epidemic diseases induced by the coronaviruses, is the respiratory transmission (Kahn and McIntosh, 2005; Hui and Zumla, 2019; Tang et al., 2020; Annas et al., 2020; Boukanjime et al., 2020). Hence, keeping the essential contacting distance between the healthy groups and the infected groups is very important for controlling the COVID-19. contacting distance refers to the extent to which people experience a sense of familiarity (nearness and intimacy) or unfamiliarity (farness and difference) between themselves and people belonging to different social, ethnic, occupational, and religious groups from their own (Bogardus, 1925; Hodgetts et al., 2011; Ma et al., 2020). the contacting distance describes the distance between different groups, and this notion includes all differences such as social class, race/ethnicity or sexuality, but also the fact that the different groups do not mix (Bogardus, 1925; Hodgetts et al., 2011). Therefore, in the mathematically modelling processes, the contacting distance between the healthy groups and the symptomatic infected ones is a critical parameter which determines the control measures for the COVID-19. Motivated by these, this paper will present an SEIR-type model incorporating the contacting distance between 
4 Zhihui Ma, Shufan Wang, Xuanru Lin, Xiaohua Li, Xiaotao Han, Haoyang Wang, Hua Liu

the healthy groups and the infected groups, and mainly focus on exploiting the control measures for the COVID-19 based on the contacting distance. This paper will determine the threshold contacting distance between the healthy groups and the infected groups, and the COVID-19 will be ultimately controlled when the contacting distance between the healthy groups and the infected groups is larger than this threshold contacting distance. Otherwise, the COVID-19 may not be extinct. Here, the threshold contacting distance is a critical value, and the COVID-19 will be extinct under this threshold contacting distance and the COVID-19 may be endemic above this threshold contacting distance.

\section{Method}

In this section, an epidemic SEIR-type model with the contacting distance between the healthy groups and the symptomatic infected groups are presented to describe the spread of the COVID-19 based the following two steps.

Step 1. Proposing an SEIR-type epidemic model for the COVID-19 with the incidence rate which will be presented in the later step 2 .

$\left(A_{11}\right)$ Based on the spreading mechanism of the COVID-19, the total populations are divided into five subclasses: the susceptible subpopulation $(S(t))$, the incubation subpopulation $(E(t))$, the asymptomatic infected subpopulation $\left(I_{A}(t)\right)$, the symptomatic infected subpopulation $(I(t))$ and the recovered subpopulation $(R(t))$.

$\left(A_{12}\right)$ The mean migrating rate of susceptible population for a local region, which describes the local outdoor-movements of the susceptible individuals during the spread of the COVID-19, is a constant recruitment $A$, which is defined in the interval $[0,+\infty)$. If the mean migrating rate is larger than zero and smaller than one, it means that individuals can only immigrate into the objective streets from their own home in their own local region. If the mean migrating rate is larger than one, it implies that some parts of individuals immigrate to the objective streets of the local region from the other regions.

$\left(A_{13}\right)$ The natural death rates of all subpopulation are proportional to their existing densities, and the proportional coefficient is $\mu$. The death rates of the asymptomatic infected subpopulation and symptomatic infected subpopulation are proportional to their existing densities, and the proportional coefficients are $\mu_{21}$ and $\mu_{22}$, respectively. The death rates of the incubation subpopulation could be omitted according to the report of the 
National Health Commission of the People's Republic of China (NHCPRC) and the World Health Organization (WHO).

$\left(A_{14}\right)$ The conversion rates from the incubation subpopulation to the asymptomatic infected subpopulation and the symptomatic infected subpopulation are proportional to the former's density and the proportional coefficients are $\gamma_{11}$ and $\gamma_{12}$, respectively. These conversion rates are mainly determined by the incubation period of the COVID-19.

$\left(A_{15}\right)$ The recovery rate of the asymptomatic infected subpopulation and symptomatic infected subpopulation are proportional to their densities, and the proportional coefficients are $\gamma_{21}$ and $\gamma_{22}$, respectively. These are mainly determined by their autoimmunity and the medical level of the local hospitals. At present, the recovery rate of the symptomatic infected individuals prominently increase under the effective medical measures being adopted by the local government.

$\left(A_{16}\right)$ The conversion rates from the susceptible subpopulation to the asymptomatic infected subpopulation and the asymptomatic infected subpopulation are defined as the incidence rate which are proposed in the following step 2.

Step 2. According to our published work (Ma et al., 2020) which was done by some authors of this paper, we present the two incidence functions with the contacting distance explicitly for the asymptomatic and symptomatic infected populations induced by the COVID-19 as the following form, respectively.

$\left(I F\left(I_{A}\right)\right)$ The incidence functions with the contacting distance explicitly for the asymptomatic infected populations

$$
I F\left(I_{A}\right)=\frac{\beta_{1}(1-d) S I_{A}}{1+\beta_{1}(1-d) h_{1} I_{A}},
$$

where $\beta_{1}$ is the natural infectious rate of the asymptomatic infected individuals who are carrying the coronavirus-2019, $h_{1}$ is the average effective contacting time of each asymptomatic infected individual carrying the coronavirus-2019.

Again, the term $\beta_{1}(1-d) I_{A}$ measures the positive infection force of the COVID-19 for each susceptible individual, and the term $\frac{1}{1+\beta_{1}(1-d) h_{1} I_{A}}$ describes the inhibition effect when the number of the asymptomatic infected individuals increases. At present, this inhibition effect for the COVID-19 are always existing since peoples are wearing the gauze masks and staying away from the crowds as far as possible under the media effect and fear effect for the COVID-19. It is obtained that the term $\lim _{I_{A} \rightarrow+\infty} \frac{N\left(I_{A}\right)}{T}=\frac{1}{h_{1}}$ 
6 Zhihui Ma, Shufan Wang, Xuanru Lin, Xiaohua Li, Xiaotao Han, Haoyang Wang, Hua Liu

is constant, in other words, the infectious ability of the COVID-19 will be saturated when the number of the asymptomatic infected individuals is large enough since the the asymptomatic infected individuals are quarantined .

$(I F(I))$ The incidence functions with the contacting distance explicitly for the symptomatic infected populations

$$
I F(I)=\frac{\beta_{2}(1-d) S I}{1+\beta(1-d) h_{2} I},
$$

where $\beta_{2}$ is the intrinsically infectious rate of the symptomatic individuals infected by the COVID-19, $h_{2}$ is the average outdoor-time of each symptomatic individuals infected by the COVID-19.

It is reasonable to assumed that $\beta_{1}<\beta_{2}$ and $h_{1}<h_{2}$.

Based on the above two steps, the epidemic model for the COVID-19 incorporating the contacting distance is presented as the following form:

$$
\left\{\begin{array}{l}
\dot{S}(t)=A-\frac{\beta_{1}(1-d) S I_{A}}{1+\beta_{1}(1-d) h_{1} I_{A}}-\frac{\beta_{2}(1-d) S I}{1+\beta_{2}(1-d) h_{2} I}-\mu S \\
\dot{E}(t)=\frac{\beta_{1}(1-d) S I_{A}}{1+\beta_{1}(1-d) h_{1} I_{A}}+\frac{\beta_{2}(1-d) S I}{1+\beta_{2}(1-d) h_{2} I}-\mu E-\mu_{1} E-\gamma_{11} E-\gamma_{12} E \\
\dot{I_{A}}(t)=\gamma_{11} E-\mu I_{A}-\mu_{21} I_{A}-\gamma_{21} I_{A} \\
\dot{I}(t)=\gamma_{12} E-\mu I-\mu_{22} I-\gamma_{22} I \\
\dot{R}(t)=\mu_{1} E+\gamma_{21} I_{A}+\gamma_{22} I-\mu R
\end{array}\right.
$$

with the initial conditions:

$$
S(0) \geq 0, \quad E(0) \geq 0, \quad I_{A}(0) \geq 0, \quad I(0) \geq 0, \quad R(0) \geq 0 .
$$

Note that the recover variable $R(t)$ does not appear in the first four equations of model (2.5), this paper focuses on the following subsystem which mainly determine the dynamical behaviors of the original model system:

$$
\left\{\begin{array}{l}
\dot{S}(t)=A-\frac{\beta_{1}(1-d) S I_{A}}{1+\beta_{1}(1-d) h_{1} I_{A}}-\frac{\beta_{2}(1-d) S I}{1+\beta_{2}(1-d) h_{2} I}-\mu S \\
\dot{E}(t)=\frac{\beta_{1}(1-d) S I_{A}}{1+\beta_{1}(1-d) h_{1} I_{A}}+\frac{\beta_{2}(1-d) S I}{1+\beta_{2}(1-d) h_{2} I}-\mu E-\mu_{1} E-\gamma_{11} E-\gamma_{12} E \\
\dot{I_{A}}(t)=\gamma_{11} E-\mu I_{A}-\mu_{21} I_{A}-\gamma_{21} I_{A} \\
\dot{I}(t)=\gamma_{12} E-\mu I-\mu_{22} I-\gamma_{22} I
\end{array}\right.
$$

with the initial conditions:

$$
S(0) \geq 0, \quad E(0) \geq 0, \quad I_{A}(0) \geq 0, \quad I(0) \geq 0 .
$$




\section{Results}

\subsection{Basic property}

In order to guarantee the ecologically and epidemically realistic meanings of model (2.7) and model (2.8) with their own initial conditions, the positivity and boundness of all solutions of model (2.7) and model (2.8) with their own initial conditions should be validated.

Proposition 3.1.1. All solutions ( $\left.S(t), E(t), I_{A}(t), I(t), R(t)\right)$ of model (2.7) with the initial conditions (2.8) are positive on the finite region for all $t>0$.

Proposition 3.1.2. All solutions $\left(S(t), E(t), I_{A}(t), I(t), R(t)\right)$ of model (2.7) with the initial conditions (2.8) are bounded on the finite region in the following set $\Omega$, where

$$
\left.\left.\Omega=\left\{\left(S(t), E(t), I_{A}(t), I(t)\right), R(t)\right) \in R_{+}^{5} \mid 0<S(t)+E(t)+I_{A}(t)+I(t)\right)+R(t) \leq \frac{A}{\mu}\right\} .
$$

Based on the above theorems, the presented model (2.7) with the initial conditions (2.8) is mathematically well-behaviored, and has its own epidemiological and ecological meanings. Hence, the realistic aspects and properties of model (2.7) with the initial conditions (2.8) are also guaranteed. Next, this paper focuses on the dynamical behaviors of model (2.7) with the initial conditions (2.8) and the theoretically controlling methods for the epidemic diseases.

\subsection{The basic reproduction number}

Let

$$
F=\left(\begin{array}{c}
\frac{\beta_{1}(1-d) S I_{A}}{1+\beta_{1}(1-d) h_{1} I_{A}}+\frac{\beta_{2}(1-d) S I}{1+\beta_{2}(1-d) h_{2} I} \\
0 \\
0 \\
0 \\
0
\end{array}\right)
$$

and

$$
V=\left(\begin{array}{c}
\frac{\beta_{1}(1-d) S I_{A}}{1+\beta_{1}(1-d) h_{1} I_{A}}+\frac{\beta_{2}(1-d) S I}{1+\beta_{2}(1-d) h_{2} I} \\
\left(\mu+\mu_{1}+\gamma_{11}+\gamma_{12}\right) E \\
\left(\mu+\mu_{21}+\gamma_{21}\right) I_{A}-\gamma_{11} E \\
\mu R-\mu_{1} E+\gamma_{21} I_{A}-\gamma_{22} I \\
\frac{\beta_{1}(1-d) S I_{A}}{1+\beta_{1}(1-d) h_{1} I_{A}}+\frac{\beta_{2}(1-d) S I}{1+\beta_{2}(1-d) h_{2} I}+\mu S-A
\end{array}\right)
$$


8 Zhihui Ma, Shufan Wang, Xuanru Lin, Xiaohua Li, Xiaotao Han, Haoyang Wang, Hua Liu

Thus, it is obtained that

$$
\left.D F\right|_{P_{0}}=\left(\begin{array}{ccccc}
0 & \frac{\beta_{1}(1-d) A}{\mu} & \frac{\beta_{2}(1-d) A}{\mu} & 0 & 0 \\
0 & 0 & 0 & 0 & 0 \\
0 & 0 & 0 & 0 & 0 \\
0 & 0 & 0 & 0 & 0 \\
0 & 0 & 0 & 0 & 0
\end{array}\right),
$$

and

$$
\left.D V\right|_{P_{0}}=\left(\begin{array}{ccccc}
\mu+\mu_{1}+\gamma_{11}+\gamma_{12} & 0 & 0 & 0 & 0 \\
-\gamma_{11} & \mu+\mu_{21}+\gamma_{21} & 0 & 0 & 0 \\
-\gamma_{12} & 0 & \mu+\mu_{22}+\gamma_{22} & 0 & 0 \\
-\mu_{1} & -\gamma_{21} & -\gamma_{22} & \mu & 0 \\
0 & \frac{\beta_{1}(1-d) A}{\mu} & \frac{\beta_{2}(1-d) A}{\mu} & 0 & \mu
\end{array}\right) .
$$

Thus, we have

$$
\left(\left.D V\right|_{P_{0}}\right)^{-1}=\left(\begin{array}{ccccc}
\frac{1}{\mu+\mu_{1}+\gamma_{11}+\gamma_{12}} & 0 & 0 & 0 & 0 \\
\frac{1}{\left(\mu+\mu_{1}+\gamma_{11}+\gamma_{12}\right)\left(\mu+\mu_{21}+\gamma_{21}\right)} & \frac{1}{\mu+\mu_{21}+\gamma_{21}} & 0 & 0 & 0 \\
\frac{1}{\left(\mu+\mu_{1}+\gamma_{11}+\gamma_{12}\right)\left(\mu+\mu_{22}+\gamma_{22}\right)} & 0 & \frac{1}{\mu+\mu_{22}+\gamma_{22}} & 0 & 0 \\
a_{41} & \frac{\gamma_{21}}{\mu\left(\mu+\mu_{21}+\gamma_{21}\right)} & \frac{\gamma_{22}}{\mu\left(\mu+\mu_{22}+\gamma_{22}\right)} & \frac{1}{\mu} & 0 \\
a_{51} & \frac{\beta_{1}(1-d) A}{\mu\left(\mu+\mu_{21}+\gamma_{21}\right)} & \frac{\beta_{2}(1-d) A}{\mu\left(\mu+\mu_{22}+\gamma_{22}\right)} & 0 & \frac{1}{\mu}
\end{array}\right),
$$

in which

$$
\left\{\begin{array}{l}
a_{41}=\frac{\mu \mu_{1}\left(\mu+\mu_{21}+\gamma_{21}\right)\left(\mu+\mu_{22}+\gamma_{22}\right)+\mu \gamma_{11} \gamma_{21}\left(\mu+\mu_{22}+\gamma_{22}\right)+\mu \gamma_{12} \gamma_{22}\left(\mu+\mu_{21}+\gamma_{21}\right)}{|D V|}>0, \\
a_{51}=-\frac{\beta_{1}(1-d) A \gamma_{11}\left(\mu+\mu_{22}+\gamma_{22}\right)+\beta_{2}(1-d) A \gamma_{12}\left(\mu+\mu_{21}+\gamma_{21}\right)}{|D V|}<0 .
\end{array}\right.
$$

Therefore, the basic reproduction number is

$$
R_{0}=\rho\left(\left(\left.D F\right|_{P_{0}}\right) \bullet\left(\left.D V\right|_{P_{0}}\right)^{-1}\right)=\frac{(1-d) A}{\mu\left(\mu+\mu_{1}+\gamma_{11}+\gamma_{12}\right)}\left(\frac{\beta_{1} \gamma_{11}}{\mu+\mu_{21}+\gamma_{21}}+\frac{\beta_{2} \gamma_{12}}{\mu+\mu_{22}+\gamma_{22}}\right) .
$$

\subsection{The extinct threshold value}

In this section, the importantly interesting thing is to consider the dynamical behavior of the disease-free equilibrium since the main focus is to develop the controlling methods for the COVID19. 
Clearly, the disease-free equilibrium point of model (2.7) $P_{0}$ is $\left(\frac{A}{\mu}, 0,0,0\right)$, and we mainly consider the dynamical behavior of the disease-free equilibrium point $E_{0}$.

The Jacobia matrix of model $(2.7)$ at the disease-free equilibrium point $P_{0}$ is as follows

$$
J_{P_{0}}=\left(\begin{array}{cccc}
-\mu & 0 & -\frac{A_{4}(1-d)}{\gamma_{11}} & -\frac{A_{5}(1-d)}{\gamma_{12}} \\
0 & -A_{1} & \frac{A_{4}(1-d)}{\gamma_{11}} & \frac{A_{5}(1-d)}{\gamma_{12}} \\
0 & \gamma_{11} & -A_{2} & 0 \\
0 & \gamma_{12} & 0 & -A_{3}
\end{array}\right)
$$

where

$$
\left\{\begin{array}{l}
A_{1}=\mu+\mu_{1}+\gamma_{11}+\gamma_{12}>0 \\
A_{2}=\mu+\mu_{21}+\gamma_{21}>0 \\
A_{3}=\mu+\mu_{22}+\gamma_{22}>0 \\
A_{4}=\frac{\beta_{1} \gamma_{11} A}{\mu}>0 \\
A_{5}=\frac{\beta_{2} \gamma_{12} A}{\mu}>0
\end{array}\right.
$$

Therefore, the characteristic equation of model (2.7) at the at the disease-free equilibrium point $P_{0}$ is presented by the following form:

$$
(\lambda+\mu)\left(\lambda^{3}+B_{1} \lambda^{2}+B_{2} \lambda+B_{3}\right)=0,
$$

in which

$$
\left\{\begin{array}{l}
B_{1}=A_{1}+A_{2}+A_{3}>0 \\
B_{2}=A_{1} A_{2}+A_{1} A_{3}+A_{2} A_{3}-A_{4}(1-d)-A_{5}(1-d), \\
B_{3}=A_{1} A_{2} A_{3}-A_{3} A_{4}(1-d)-A_{2} A_{5}(1-d) .
\end{array}\right.
$$

According to Routh-Hurwitz Rule, the disease-free equilibrium point is $P_{0}\left(\frac{A}{\mu}, 0,0,0\right)$ is locally asymptotically stable if and only if

$$
\left\{\begin{array}{l}
B_{1}>0, B_{3}>0, \\
B_{1} B_{2}-B_{3}>0 .
\end{array}\right.
$$


10 Zhihui Ma, Shufan Wang, Xuanru Lin, Xiaohua Li, Xiaotao Han, Haoyang Wang, Hua Liu

Next, two cases are considered:

$$
\begin{aligned}
& B_{3}>0 \\
& \Leftrightarrow A_{1} A_{2} A_{3}-\left(A_{3} A_{4}+A_{2} A_{5}\right)(1-d)>0 \\
& \Leftrightarrow \frac{1-d}{A_{1}}\left(\frac{A_{4}}{A_{2}}+\frac{A_{5}}{A_{3}}\right)<1 \\
& \Leftrightarrow \frac{(1-d) A}{\mu\left(\mu+\mu_{1}+\gamma_{11}+\gamma_{12}\right)}\left(\frac{\beta_{1} \gamma_{11}}{\mu+\mu_{21}+\gamma_{21}}+\frac{\beta_{2} \gamma_{12}}{\mu+\mu_{22}+\gamma_{22}}\right)<1 \\
& \Leftrightarrow R_{0}<1
\end{aligned}
$$

Again, we have

$$
\begin{aligned}
B_{1} & B_{2}-B_{3}>0 \\
\Leftrightarrow & \left(A_{1}+A_{2}+A_{3}\right)\left(A_{1} A_{3}+A_{2} A_{3}+A_{1} A_{2}-A_{4}(1-d)-A_{5}(1-d)\right) \\
& -\left(A_{1} A_{2} A_{3}-A_{3} A_{4}(1-d)-A_{2} A_{5}(1-d)\right)>0 \\
\Leftrightarrow & A_{1}^{2} A_{3}+2 A_{1} A_{2} A_{3}+A_{1}^{2} A+A_{2}^{2} A_{3}+A_{1} A_{2}^{2}+A_{1} A_{3}^{2}+A_{2} A_{3}^{2} \\
& -\left[\left(A_{1}+A_{2}\right) A_{4}+\left(A_{1}+A_{3}\right) A_{5}\right](1-d)>0 \\
\Leftrightarrow & \left(A_{1}+A_{3}\right)\left(A_{1} A_{3}+A_{2}\left(A_{1}+A_{2}+A_{3}\right)\right) \\
& -\left[\left(A_{1}+A_{2}\right) A_{4}+\left(A_{1}+A_{3}\right) A_{5}\right](1-d)>0 \\
\Leftrightarrow & 2 A_{1} A_{2} A_{3}+A_{1} A_{3}^{2}+A_{2}^{2} A_{3}+A_{2} A_{3}^{2}+A_{1} A_{2}^{2} \\
& -\left(A_{1} A_{4}(1-d)+A_{1} A_{5}(1-d)\right)-\left(A_{2} A_{4}(1-d)+A_{3} A_{5}(1-d)\right)>0 .
\end{aligned}
$$

If $R_{0}<1$, then we have

$$
\begin{aligned}
& 2 A_{1} A_{2} A_{3}+A_{1} A_{3}^{2}+A_{2}^{2} A_{3}+A_{2} A_{3}^{2}+A_{1} A_{2}^{2} \\
& -\left(A_{1} A_{4}(1-d)+A_{1} A_{5}(1-d)\right)-\left(A_{2} A_{4}(1-d)+A_{3} A_{5}(1-d)\right) \\
& >\left(A_{1}+A_{2}\right)\left(A_{1}+A+3\right)>0 .
\end{aligned}
$$

Therefore, the disease-free equilibrium point is $P_{0}\left(\frac{A}{\mu}, 0,0,0\right)$ is locally asymptotically stable if and only if

$$
R_{0}<1 \Leftrightarrow d>1-\frac{\mu\left(\mu+\mu_{1}+\gamma_{11}+\gamma_{12}\right)\left(\mu+\mu_{22}+\gamma_{22}\right)\left(\mu+\mu_{21}+\gamma_{21}\right)}{\beta_{1} \gamma_{11} A\left(\mu+\mu_{22}+\gamma_{22}\right)+\beta_{2} \gamma_{12} A\left(\mu+\mu_{21}+\gamma_{21}\right)}>0
$$

Based on the above analysis, we obtain the following results.

Proposition 3.2.1. Suppose that $\beta_{1} \gamma_{11} A\left(\mu+\mu_{22}+\gamma_{22}\right)+\beta_{2} \gamma_{12} A\left(\mu+\mu_{21}+\gamma_{21}\right)>\mu\left(\mu+\mu_{1}+\right.$ $\left.\gamma_{11}+\gamma_{12}\right)\left(\mu+\mu_{22}+\gamma_{22}\right)\left(\mu+\mu_{21}+\gamma_{21}\right)$, we have

(1). If $R_{0}>1$ or $d<1-\frac{\mu\left(\mu+\mu_{1}+\gamma_{11}+\gamma_{12}\right)\left(\mu+\mu_{22}+\gamma_{22}\right)\left(\mu+\mu_{21}+\gamma_{21}\right)}{\beta_{1} \gamma_{11} A\left(\mu+\mu_{22}+\gamma_{22}\right)+\beta_{2} \gamma_{12} A\left(\mu+\mu_{21}+\gamma_{21}\right)}$, then the diseasefree equilibrium point $P_{0}$ is unstable, 
(2). If $R_{0}<1$ or $d>1-\frac{\mu\left(\mu+\mu_{1}+\gamma_{11}+\gamma_{12}\right)\left(\mu+\mu_{22}+\gamma_{22}\right)\left(\mu+\mu_{21}+\gamma_{21}\right)}{\beta_{1} \gamma_{11} A\left(\mu+\mu_{22}+\gamma_{22}\right)+\beta_{2} \gamma_{12} A\left(\mu+\mu_{21}+\gamma_{21}\right)}$, then the diseasefree equilibrium point $P_{0}$ is locally asymptotically stable.

Proposition 3.2.1 reveals that the COVID-19 can be controlled while the contacting distance between the healthy individuals and the symptomatic infected individuals is larger than the threshold value $d^{*}=1-\frac{\mu\left(\mu+\mu_{1}+\gamma_{11}+\gamma_{12}\right)\left(\mu+\mu_{22}+\gamma_{22}\right)\left(\mu+\mu_{21}+\gamma_{21}\right)}{\beta_{1} \gamma_{11} A\left(\mu+\mu_{22}+\gamma_{22}\right)+\beta_{2} \gamma_{12} A\left(\mu+\mu_{21}+\gamma_{21}\right)}$. This threshold value decreases with the decrease in the immigration rate of susceptible population.

\subsection{Model (2.7) without the asymptomatic infected population}

If the asymptomatic infected population is omitted since it is difficult to be found and the infection probability of the asymptomatic individuals is very small, then the model (2.7) will become the following forms

$$
\left\{\begin{array}{l}
\dot{S}(t)=A-\frac{\beta_{2}(1-d) S I}{1+\beta_{2}(1-d) h_{2} I}-\mu S \\
\dot{E}(t)=\frac{\beta_{2}(1-d) S I}{1+\beta_{2}(1-d) h_{2} I}-\mu E-\mu_{1} E-\gamma_{12} E \\
\dot{I}(t)=\gamma_{12} E-\mu I-\mu_{22} I-\gamma_{22} I
\end{array}\right.
$$

with the initial conditions:

$$
S(0) \geq 0, \quad E(0) \geq 0, \quad I(0) \geq 0 .
$$

According to Proposition 3.1.1 and Proposition 3.1.2, all solutions of model (3.11) with the initial conditions (3.12) are positive and bounded.

According to the computation of the basic reproduction number of model (2.7) $\tilde{R}_{0}$, the basic reproduction number of model(3.11) is

$$
\tilde{R}_{0}=\frac{\beta_{2} \gamma_{12} A(1-d)}{\mu\left(\mu+\mu_{1}+\gamma_{12}\right)\left(\mu+\mu_{22}+\gamma_{22}\right)},
$$

By simple computation, the disease-free equilibrium point of model $(3.11)$ is $\tilde{P}_{0}\left(\frac{A}{\mu}, 0,0\right)$ and the corresponding endemic equilibrium point is $\tilde{P}(\tilde{S}, \tilde{E}, \tilde{I})$, where

$$
\left\{\begin{array}{l}
\tilde{S}=\frac{\beta_{2} \gamma_{12}(1-d)+\mu\left(\mu+\mu_{1}+\gamma_{12}\right)\left(\mu+\mu_{22}+\gamma_{22}\right)}{\beta_{2} \gamma_{12}(1-d)\left(\mu h_{2}+1\right)}, \\
\tilde{E}=\frac{\beta_{2} \gamma_{12}(1-d)-\mu\left(\mu+\mu_{1}+\gamma_{12}\right)\left(\mu+\mu_{22}+\gamma_{22}\right)}{\beta_{2} \gamma_{12}(1-d)\left(\mu h_{2}+1\right)\left(\mu+\mu_{1}+\gamma_{12}\right)} \\
\tilde{I}=\frac{\beta_{2} \gamma_{12}(1-d)-\mu\left(\mu+\mu_{1}+\gamma_{12}\right)\left(\mu+\mu_{22}+\gamma_{22}\right)}{\beta_{2} \gamma_{12}(1-d)\left(\mu h_{2}+1\right)\left(\mu+\mu_{1}+\gamma_{12}\right)\left(\mu+\mu_{22}+\gamma_{22}\right)}
\end{array} .\right.
$$

Clearly, the endemic equilibrium point $\tilde{P}$ is positive if and only if $\tilde{R}_{0}>1$. 
12 Zhihui Ma, Shufan Wang, Xuanru Lin, Xiaohua Li, Xiaotao Han, Haoyang Wang, Hua Liu

Next, the stability properties of the disease-free equilibrium point $\tilde{P}_{0}$ and the endemic equilibrium point $\tilde{P}$ are considered.

Firstly, the Jacobia matrix of model (3.11) at the disease-free equilibrium point $\bar{P}_{0}$ is as follows

$$
J_{\tilde{P}_{0}}=\left(\begin{array}{ccc}
-\mu & 0 & -\frac{\beta_{2} A(1-d)}{\mu} \\
0 & -A_{1} & \frac{\beta_{2} A(1-d)}{\mu} \\
0 & \gamma_{12} & -A_{3}
\end{array}\right) .
$$

The corresponding characteristic equation of model (3.11) at the disease-free equilibrium point $\tilde{P}_{0}$ is

$$
(\lambda+\mu)\left(\lambda^{2}+\left(A_{1}+A_{3}\right) \lambda+A_{1} A_{3}-A_{5}(1-d)\right)
$$

Therefore, according to Routh-Hurwitz Rule, the disease-free equilibrium point is $\tilde{P}_{0}$ is locally asymptotically stable if and only if

$$
\begin{aligned}
& A_{1} A_{3}-A_{5}(1-d)>0 \\
& \Leftrightarrow \frac{A_{5}(1-d)}{A_{1} A_{3}}<1 \\
& \Leftrightarrow \tilde{R}_{0}<1 \\
& \Leftrightarrow d>1-\frac{\mu\left(\mu+\mu_{1}+\gamma_{12}\right)\left(\mu+\mu_{22}+\gamma_{22}\right)}{\beta_{2} A \gamma_{12}}>0 .
\end{aligned}
$$

Secondly, the Jacobia matrix of model (3.11) at the endemic equilibrium point $\tilde{P}$ is as follows

$$
J_{\tilde{P}}=\left(\begin{array}{ccc}
-\mu-\frac{\beta_{2} A(1-d) \tilde{I}}{1+\beta_{2}(1-d) h_{2} \tilde{I}} & 0 & \frac{\beta_{2}(1-d)\left(\left(\mu+\mu_{1}+\gamma_{12}\right)\left(\mu+\mu_{22}+\gamma_{22}\right)-\gamma_{12} A\right)}{\mu \gamma_{12}\left(1+\beta_{2}(1-d) h_{2} \tilde{I}\right)^{2}} \\
\frac{\beta_{2} A(1-d) \tilde{I}}{1+\beta_{2}(1-d) h_{2} \tilde{I}} & -\left(\mu+\mu_{1}+\gamma_{12}\right) & \frac{\beta_{2}(1-d)\left(\gamma_{12} A-\left(\mu+\mu_{1}+\gamma_{12}\right)\left(\mu+\mu_{22}+\gamma_{22}\right)\right)}{\mu \gamma_{12}\left(1+\beta_{2}(1-d) h_{2} \tilde{I}\right)^{2}} \\
0 & \gamma_{12} & -\left(\mu+\mu_{22}+\gamma_{22}\right)
\end{array}\right) .
$$

The corresponding characteristic equation of model (3.11) at the endemic equilibrium point $\tilde{P}$ is

$$
\lambda^{3}+\tilde{B}_{1} \lambda^{2}+\tilde{B}_{2} \lambda+\tilde{B}_{3}
$$

where

$$
\left\{\begin{array}{l}
\tilde{B}_{1}=\frac{\beta_{2} \gamma_{12} A(1-d)\left(\mu h_{2}+1\right)}{\beta_{2} \gamma_{12} h_{2}(1-d)+A_{1} A_{2}}+A_{1}+A_{2} \\
\tilde{B}_{2}=\frac{A_{1} A_{2} h_{2}\left(\beta_{2} \gamma_{12} A(1-d)-\mu A_{1} A_{2}\right)+\beta_{2} \gamma_{12} A(1-d)\left(\mu h_{2}+1\right)\left(A_{1}+A_{2}\right)}{\beta_{2} \gamma_{12} h_{2}(1-d)+A_{1} A_{2}} \\
\tilde{B}_{3}=\frac{A_{1} A_{2}\left(\mu h_{2}+1\right)\left(\beta_{2} \gamma_{12} A(1-d)-\mu A_{1} A_{2}\right)}{\beta_{2} \gamma_{12} h_{2}(1-d)+A_{1} A_{2}}
\end{array}\right.
$$


According to Routh - Hurwitz Rule, the endemic equilibrium point $\tilde{P}$ is locally asymptotically stable if and only if

$$
\left\{\begin{array}{l}
\tilde{B}_{1}>0, \tilde{B}_{3}>0 \\
\tilde{B}_{1} \tilde{B}_{2}-\tilde{B}_{3}>0
\end{array}\right.
$$

Clearly, the term $\tilde{B}_{1}>0$ and $\tilde{B}_{3}>0$ hold if $\tilde{R}_{0}>1$ or $d<1-\frac{\mu\left(\mu+\mu_{1}+\gamma_{12}\right)\left(\mu+\mu_{22}+\gamma_{22}\right)}{\beta_{2} A \gamma_{12}}$. Again, we have

$$
\begin{aligned}
& \tilde{B}_{1} \tilde{B}_{2}-\tilde{B}_{3}>0 \\
& \Leftrightarrow \frac{\beta_{2} \gamma_{12} A(1-d)\left(\mu h_{2}+1\right)\left(A_{1}+A_{2}\right)^{2}}{\beta_{2} \gamma_{12} A h_{2}(1-d)+A_{1} A_{2}}-\frac{A_{1} A_{2} h_{2}\left(\beta_{2} \gamma_{12} A(1-d)-\mu A_{1} A_{2}\right)}{\beta_{2} \gamma_{12} A h_{2}(1-d)+A_{1} A_{2}}>0 \\
& \Leftrightarrow \frac{\left(\mu h_{2}+1\right)\left(\gamma_{12} A(1-d)\left(A_{1}^{2}+A_{2}^{2}+A_{1} A_{2}\right)+\mu A_{1}^{2} A_{2}^{2}\right)}{\beta_{2} \gamma_{12} A h_{2}(1-d)+A_{1} A_{2}}>0 .
\end{aligned}
$$

Based on the above analysis, we obtain the following results.

Proposition 3.3.1. Suppose that $\beta_{2} A \gamma_{12}>\mu\left(\mu+\mu_{1}+\gamma_{12}\right)\left(\mu+\mu_{22}+\gamma_{22}\right)$, we have

(1). If $\tilde{R}_{0}>1$ or $d<1-\frac{\mu\left(\mu+\mu_{1}+\gamma_{12}\right)\left(\mu+\mu_{22}+\gamma_{22}\right)}{\beta_{2} A \gamma_{12}}$, then the endemic equilibrium point $\tilde{P}$ is locally asymptotically stable,

(2). If $\tilde{R}_{0}<1$ or $d>1-\frac{\mu\left(\mu+\mu_{1}+\gamma_{12}\right)\left(\mu+\mu_{22}+\gamma_{22}\right)}{\beta_{2} A \gamma_{12}}$, then the disease-free equilibrium point $\tilde{P}_{0}$ is locally asymptotically stable.

Proposition 3.3.1 reveals that the COVID-19, which incorporates the relatively high infection rate, can be controlled while the contacting distance between the healthy individuals and the symptomatic infected individuals is larger than the threshold value $\tilde{d}^{*}=1-\frac{\mu\left(\mu+\mu_{1}+\gamma_{12}\right)\left(\mu+\mu_{22}+\gamma_{22}\right)}{\beta_{2} A \gamma_{12}}$. This threshold value decreases with the decrease in the immigration rate of susceptible population. That is to say, the contacting distance could be shortened in the locations with the low population density. The equivalent results of Proposition 3.2.1, which take the immigration rate of susceptible population as the controlling parameter, can also be proposed as follows.

Proposition 3.3.2. We have the following conclusions

(1). If $0<A<\frac{\mu\left(\mu+\mu_{1}+\gamma_{12}\right)\left(\mu+\mu_{22}+\gamma_{22}\right)}{\beta_{2} \gamma_{12}(1-d)}$, then the disease-free equilibrium point $\tilde{P}_{0}$ is locally asymptotically stable,

(2). If $\frac{\mu\left(\mu+\mu_{1}+\gamma_{12}\right)\left(\mu+\mu_{22}+\gamma_{22}\right)}{\beta_{2} \gamma_{12}(1-d)}<A<1$, then the endemic equilibrium point $\tilde{P}$ is locally asymptotically stable.

Proposition 3.3.2 reveals that the COVID-19 can be controlled while the immigration rate of susceptible population is smaller than the threshold value $A^{*}=\frac{\mu\left(\mu+\mu_{1}+\gamma_{12}\right)\left(\mu+\mu_{22}+\gamma_{22}\right)}{\beta_{2}(1-d)}$, 
14 Zhihui Ma, Shufan Wang, Xuanru Lin, Xiaohua Li, Xiaotao Han, Haoyang Wang, Hua Liu

and this threshold value increases with the increase in the contacting distance between the healthy individuals and the symptomatic infected individuals. That is to say, only if the contacting distance is large enough, the relatively large immigration of local peoples can not induce the second outbreak of the COVID-19.

\subsection{Sensitivity analysis}

In this section, the numerical test for model (3.11) is conducted based on the reported cases induced by the COVID-19 in Wuhan in Hubei province and the estimated parameters of the published works (Li et al., 2020; Tang et al., 2020). All parameter values are given in the following Table 1.

Table 1. The estimated values of parameters in model (3.11)

\begin{tabular}{|c|c|c|c|}
\hline Parameter & Definitions & Mean value & Source \\
\hline$A$ & The infux of the susceptible subpopulation & Variable & \\
\hline$\beta_{2}$ & The intrinsically infectious rate & 0.8 & Li and Tang et al. \\
\hline$h_{2}$ & The average outdoor-time of each asymptomatic infected individual & 0.5 & $\mathrm{Li}$ and Tang et al. \\
\hline$\mu$ & The natural death rates of all subpopulations & 0.00711 & Li et al., Tang et al. \\
\hline$\mu_{1}$ & The recovery rate of the incubation subpopulation & 0.13978 & $\mathrm{Li}$ and Tang et al. \\
\hline$\mu_{22}$ & The death rate induced bythe coronavirus disease 2019 (COVID-19) & 0.04017 & Li and Tang et al. \\
\hline \multirow[t]{2}{*}{$\gamma_{12}$} & The transition rate from the incubation subpopulation & & \\
\hline & to the symptomatic infected subpopulation & $0.07143-0.19231$ & $\mathrm{Li}$ and Tang et al. \\
\hline$\gamma_{22}$ & The recovery rate of the symptomatic infected subpopulation & 0.33029 & Tang et al. \\
\hline \multirow[t]{2}{*}{$d$} & The contacting distance between the susceptible individuals & & \\
\hline & and the asymptomatic infected individuals & Variable & $0 \leq d \leq 1$ \\
\hline
\end{tabular}

In order to identify the key factors affecting the change of infection variables, we used Latin Hypercube Sampling (LHS) for parameter sampling and Paranoid Rank Correlation Coefficient (PRCC) method for sensitivity analysis of the parameters in the model (3.11). When performing parameter sampling, we choose uniform distribution as the prior distribution, sampling times $N=500$, the reference values of the parameters in the model are given in Table 1 , and the value range is $20 \%$ up and down the reference value. The parameters with p value greater than 0.01 are considered to have no effect on the model.

From Figure 1(a) and Table 2, it can be seen that parameter $A$ and $E(t)$ are positively correlated, that is, as parameter $A$ increases, the population of $E(t)$ subgroups will increase. $\mu_{1}, \gamma_{12}$, $d$ are negatively correlated with $E(t)$, that is, increasing $\mu_{1}, \gamma_{12}, d$ will cause $E(t)$ to decrease. It 
Table 2. $\mathbf{P R C C}$ value and $\mathbf{P}$ value of each parameter in $E(t)$

\begin{tabular}{ccc|ccc}
\hline Parameter & PRCC values & P value & Parameter & PRCC values & P value \\
\hline$A$ & 0.8300 & 0.0000 & $\gamma_{22}$ & -0.0743 & 0.3216 \\
$\mu$ & -0.0453 & 0.2541 & $\beta_{2}$ & -0.0685 & 0.9217 \\
$\mu_{1}$ & -0.5824 & 0.0000 & $h_{2}$ & 0.6829 & 0.1787 \\
$\mu_{22}$ & -0.1399 & 0.0175 & $d$ & 0.7539 & 0.0000 \\
$\gamma_{12}$ & -0.7678 & 0.0000 & & & \\
\hline
\end{tabular}

Table 3. PRCC value and $\mathbf{P}$ value of each parameter in $I(t)$

\begin{tabular}{ccc|ccc}
\hline Parameter & PRCC values & P value & Parameter & PRCC values & P value \\
\hline$A$ & 0.7808 & 0.0000 & $\gamma_{22}$ & -0.7437 & 0.0000 \\
$\mu$ & -0.1049 & 0.9927 & $\beta_{2}$ & -0.0483 & 0.7827 \\
$\mu_{1}$ & -0.4824 & 0.0000 & $h_{2}$ & 0.2149 & 0.6722 \\
$\mu_{22}$ & -0.1951 & 0.0109 & $d$ & 0.7538 & 0.0000 \\
$\gamma_{12}$ & 0.8374 & 0.0000 & & & \\
\hline
\end{tabular}

can be seen from the Figure 2 that the monotonicity of $A, \mu_{1}, \gamma_{12}, d$ is the most significant, that is, $E(t)$ is mainly affected by these parameters. Figure $1(\mathrm{~b})$ and Table 3 shows that $A$ and $\gamma_{12}$ are positively correlated with $I(t)$, that is, as $A$ and $\gamma_{12}$ increase, $I(t)$ will increase. $\mu_{1}, \gamma_{22}, d$ and $I(t)$ are negatively correlated, that is, as $\mu_{1}, \gamma_{22}, d$ increase, the population of $I(t)$ will decrease. It can be seen from the Figure 3 that the monotonicity of $A, \mu_{1}, \gamma_{12}, \gamma_{22}, d$ is the most significant, in other words, $I(t)$ is mainly affected by these parameters.

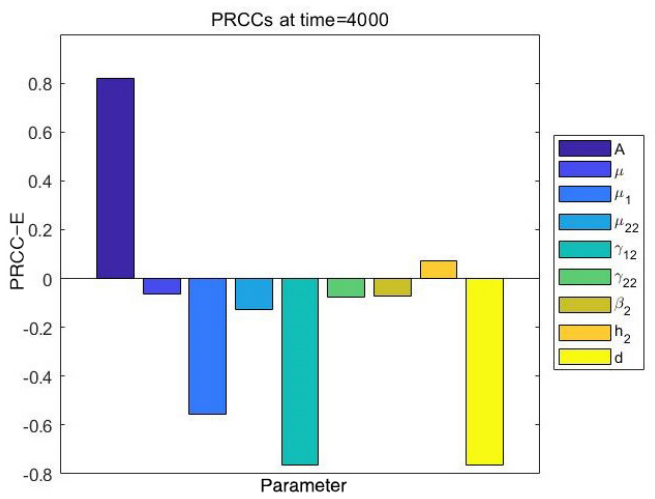

(a)

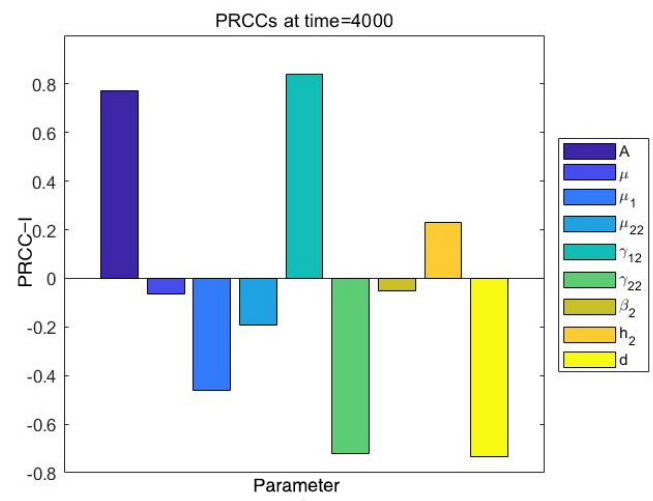

(b)

Figure 1: PRCC values of parameters in $E(t)$ and $I(t)$ at $\mathrm{t}=4000$.

It can be seen from Figure 4 that the PRCC value of each parameter changes significantly in the 
16 Zhihui Ma, Shufan Wang, Xuanru Lin, Xiaohua Li, Xiaotao Han, Haoyang Wang, Hua Liu
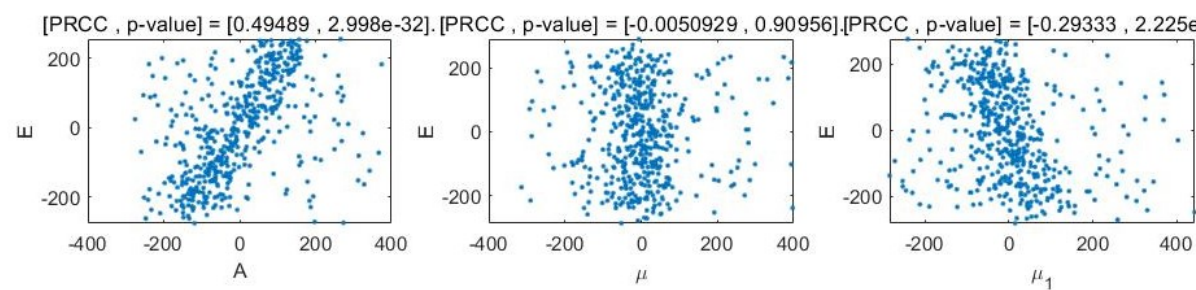

$[$ PRCC , p-value $]=[0.035245,0.43165] .[$ PRCC,$p$-value $]=[-0.41768,1.5719 \mathrm{e}-22] .[\mathrm{PRCC}, \mathrm{p}$-value $]=[-0.072476,0.10551]$
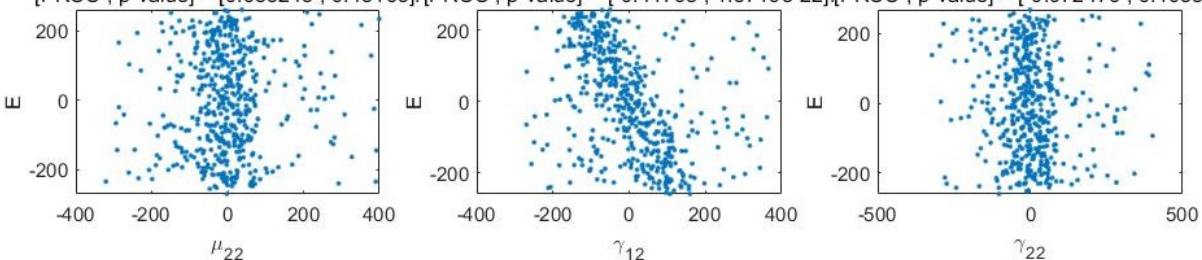

$[$ PRCC , p-value $]=[0.052498,0.24129] .[P R C C, p$-value $]=[-0.032378,0.47007] .[P R C C, p$-value $]=[-0.48945,1.761 e-31]$
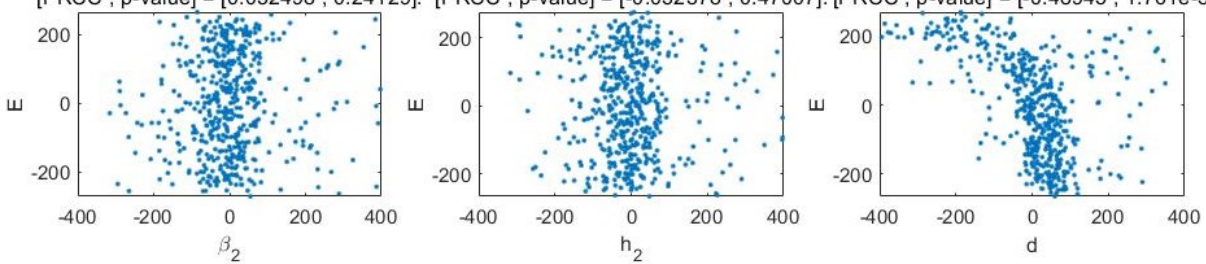

Figure 2: Scatter plot of PRCC value for each parameter in $E(t)$.
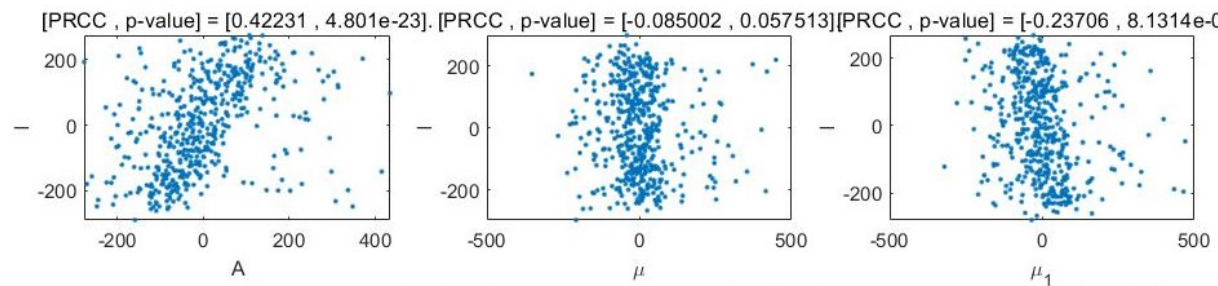

[PRCC, $p$-value $]=[-0.13221,0.0030564] \cdot[P R C C, p$-value $]=[0.46543,3.0228 \mathrm{e}-28][$ PRCC, $\mathrm{p}$-value $]=[-0.34256,3.2656 \mathrm{e}-15]$
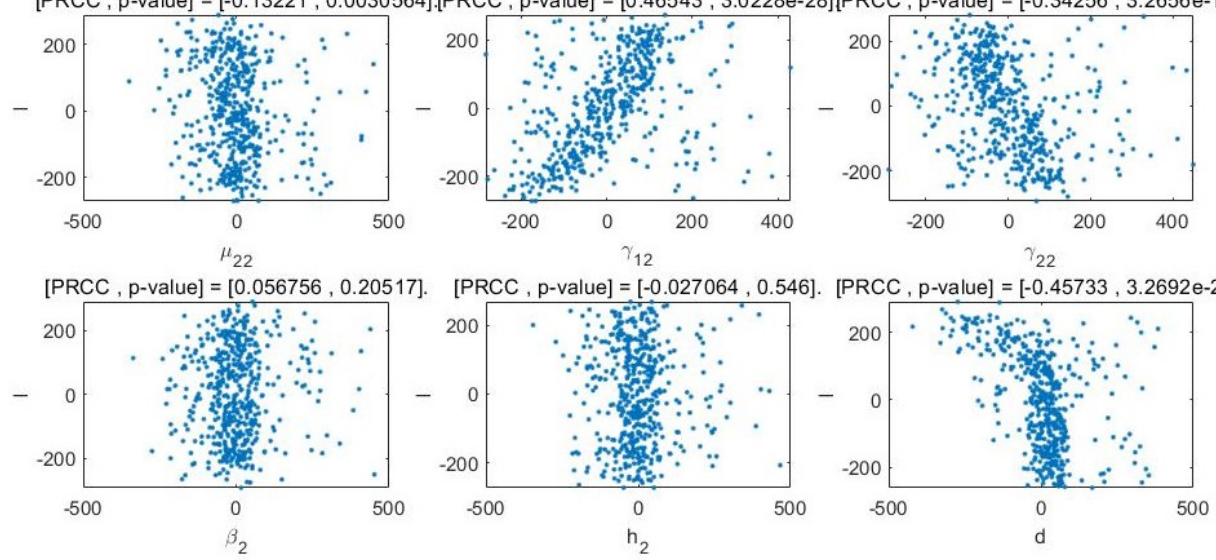

Figure 3: Scatter plot of PRCC value for each parameter in $I(t)$. 


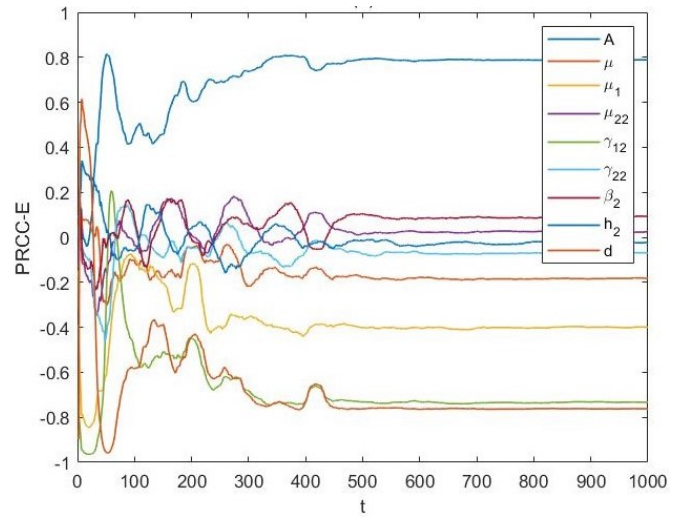

(a)

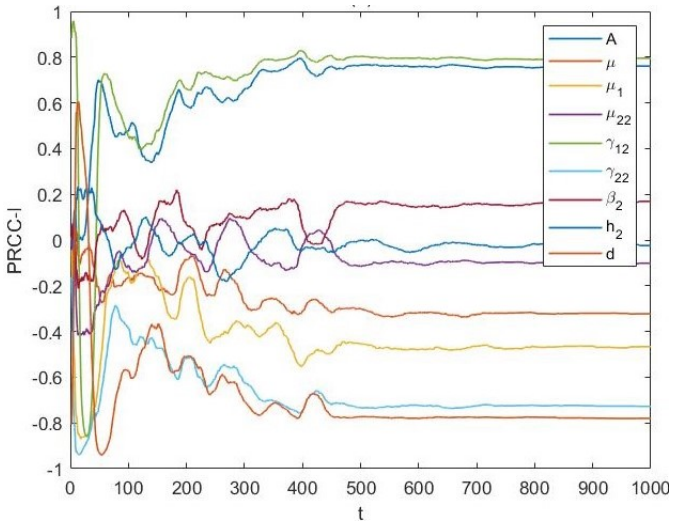

(b)

Figure 4: PRCC value of each parameter over time.

early stage of the outbreak, especially before $t=500$. It can be seen from (b) that the parameters $A, \mu_{1}, \gamma_{12}, \gamma_{22}$ and $d$ have undergone significant changes relative to $I(t)$. The PRCC values of $A$ and $d$ show a trend of first increasing, then decreasing and then increasing over time. This is due to the rapid increase in the number of patients after the outbreak, and the susceptible subpopulation $S(t)$ is more likely to be infected under the same $d$ as the initial outbreak. At this time, social distance $d$ will have a greater impact on the number of patients. With the improvement of people's awareness of the prevention of epidemics and medical conditions, people's behavior of reducing overtime and using masks will lead to less and less impact of $d$ on $I(t)$. Due to the phased results of the epidemic prevention work, people's awareness of epidemic prevention will become weaker than before. At this time, the influence of $A$ and $d$ on $I(t)$ will increase.

Table 4. PRCC value and $\mathbf{P}$ value of each parameter in $R_{0}$

\begin{tabular}{ccc|ccc}
\hline Parameter & PRCC values & P value & Parameter & PRCC values & P value \\
\hline$A$ & 0.1608 & 0.0000 & $\gamma_{22}$ & -0.1103 & 0.0000 \\
$\mu$ & -0.1737 & 0.0000 & $\beta_{2}$ & 0.1587 & 0.0000 \\
$\mu_{1}$ & -0.0517 & 0.0207 & $h_{2}$ & 0.004 & 0.8582 \\
$\mu_{22}$ & -0.0077 & 0.7307 & $d$ & -0.9094 & 0.0000 \\
$\gamma_{12}$ & 0.2112 & 0.0000 & & & \\
\hline
\end{tabular}

Figure 5 and Table 4 show the influence of each parameter on the basic reproduction number $R_{0}$. $A, \gamma_{12}, \beta_{2}$ and $R_{0}$ are positively correlated, and $\gamma_{12}$ has a greater impact on $R_{0}$. As $\gamma_{12}$ increases, $R_{0}$ will increase rapidly, and more susceptible people will be infected. Among them, $\mu, \gamma_{22}$ and $d$ are negatively correlated with $R_{0}$, and the maximum value of PRCCs is $|P R C C(d)|=0.9094$, 
18 Zhihui Ma, Shufan Wang, Xuanru Lin, Xiaohua Li, Xiaotao Han, Haoyang Wang, Hua Liu

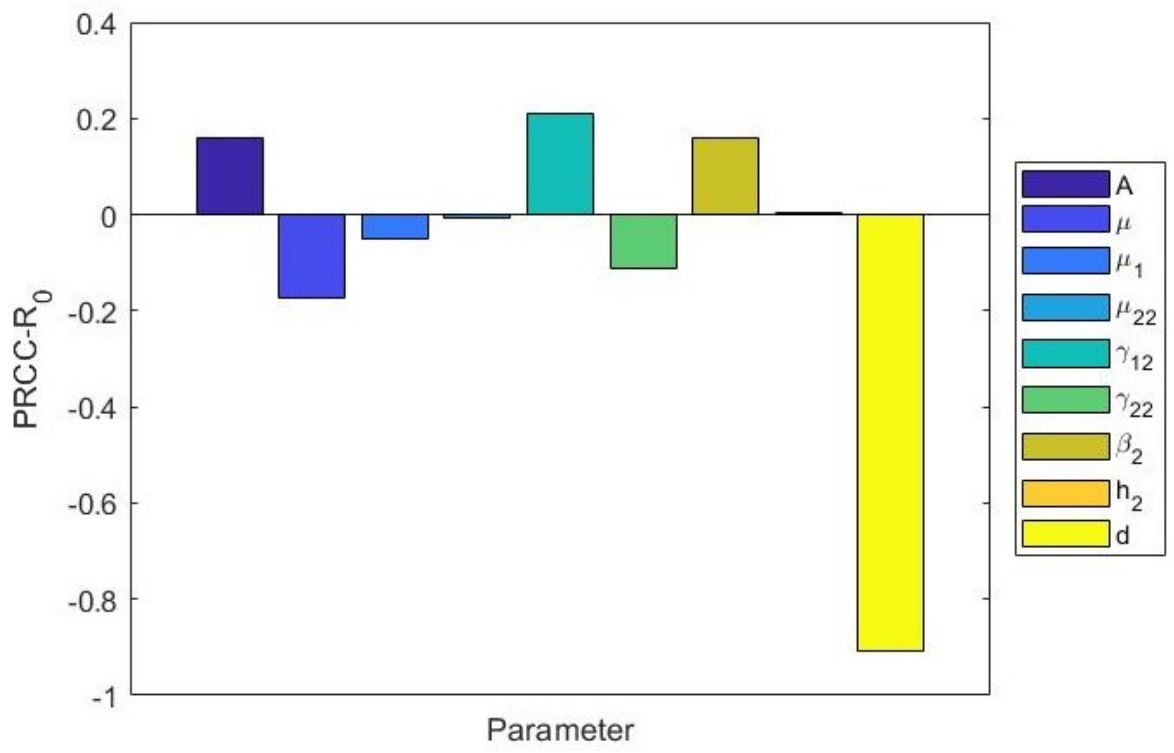

Figure 5: PRCC values of each parameter in $R_{0}$.

indicating that $d$ has the greatest impact on $R_{0}$. In other words, as long as $d$ is controlled within a certain range, $R_{0}$ can be minimized.

\subsection{Numerical test}

According to Proposition 3.3.1 and Proposition 3.3.2, the threshold values of the contacting distance $\bar{d}^{*}$ and the immigration rate $A^{*}$ are 0.8767 and 0.1849 , respectively. That is to say, the COVID-19 will be completely controlled when the contacting distance is larger than the threshold value 0.8767 , or the immigration rate smaller than the threshold value 0.1849 . Here, the immigration rate measures the density of population who immigrates to the streets from their own home in Wuhan city.

Figure 6 -Figure 8 show that the COVID-19 will be completely controlled when the contacting distance is larger than the threshold value 0.8767 . Figure 6 selects the contacting distance between the susceptible individuals and the symptomatic infected individuals as 0.89 which is larger than the threshold value 0.8767 , and hence that the COVID-19 will be vanished. Noting that the parameter of the contacting distance is normalized in the modeling processes, hence, for the COVID-19, it will be completely controlled only if the susceptible individuals and the symptomatic infected individuals keep their contacting distance to be larger than $0.8767 \times D$, where $D$ is the spread distance of the coronavirus of the COVID-19 when people are breathing and/or sneezing. 


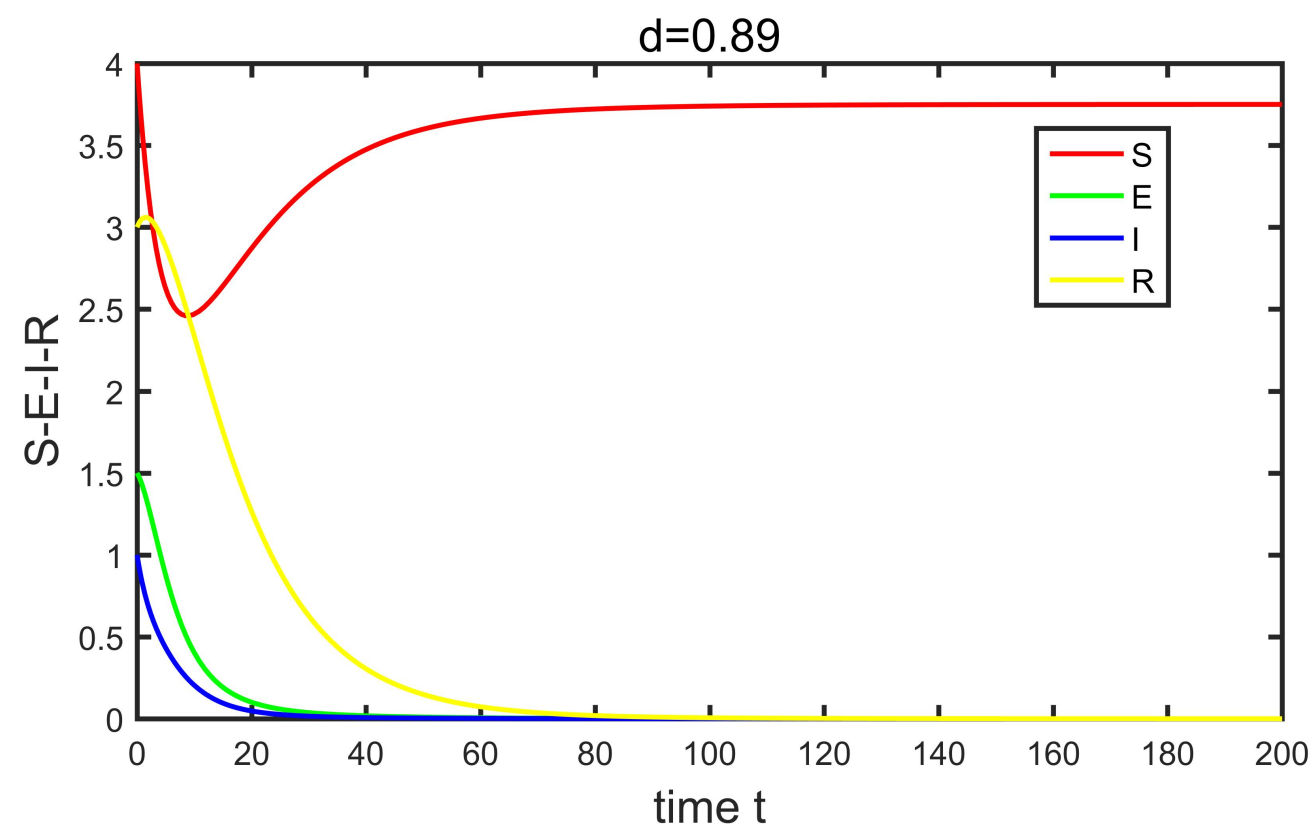

Figure 6: The disease-free equilibrium point $\bar{P}_{0}$ is globally asymptotically stable with $d=0.89$ which is larger than the threshold value 0.8767 .

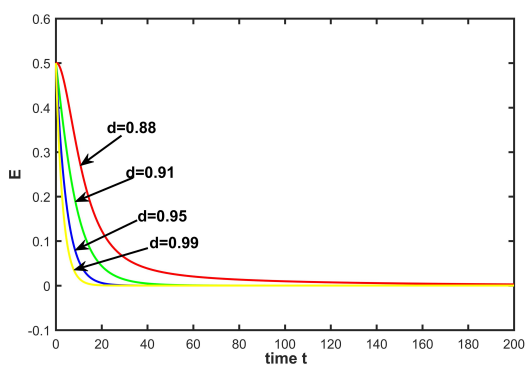

(a)

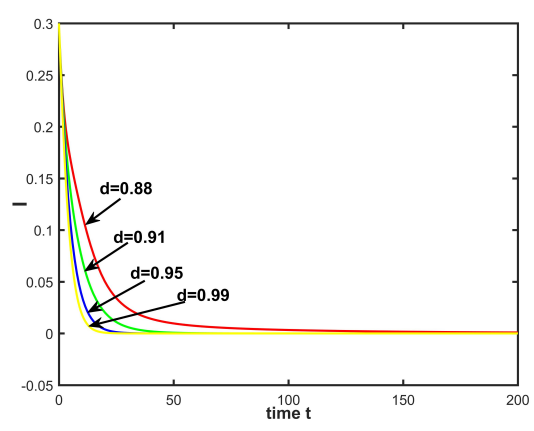

(b)

Figure 7: The time-series diagrams for $E$ and $I$ with the different contacting distances while it is larger than the threshold value 0.8767 .

Generally, individuals are require to keep away at least one meter. Furthermore, Figure 7 reveals that the extinct lag of the COVID-19 closely relates to the contacting distance when the peoples' immigrating number is 30 percent of the total population in Wuhan city after January $23^{t} h, 2020$, 
20 Zhihui Ma, Shufan Wang, Xuanru Lin, Xiaohua Li, Xiaotao Han, Haoyang Wang, Hua Liu

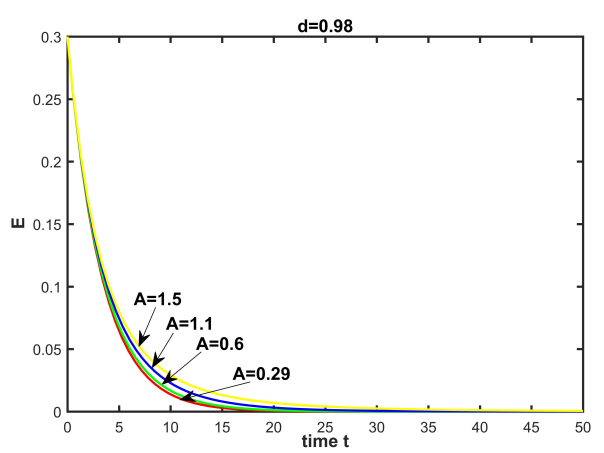

(a)

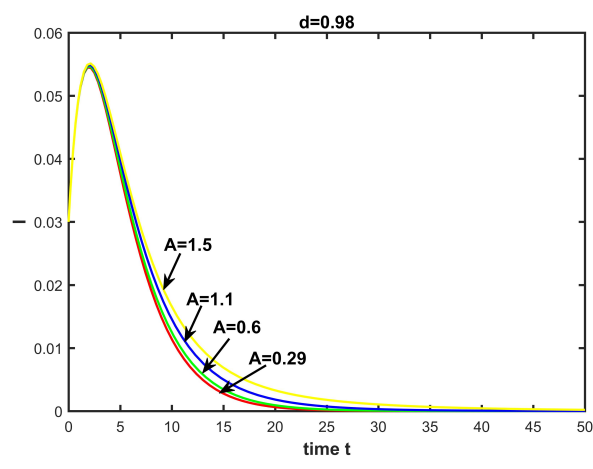

(b)

Figure 8: The time-series diagrams for $E$ and $I$ with the different immigration rates while the contacting distance is larger than the threshold value 0.8767 .

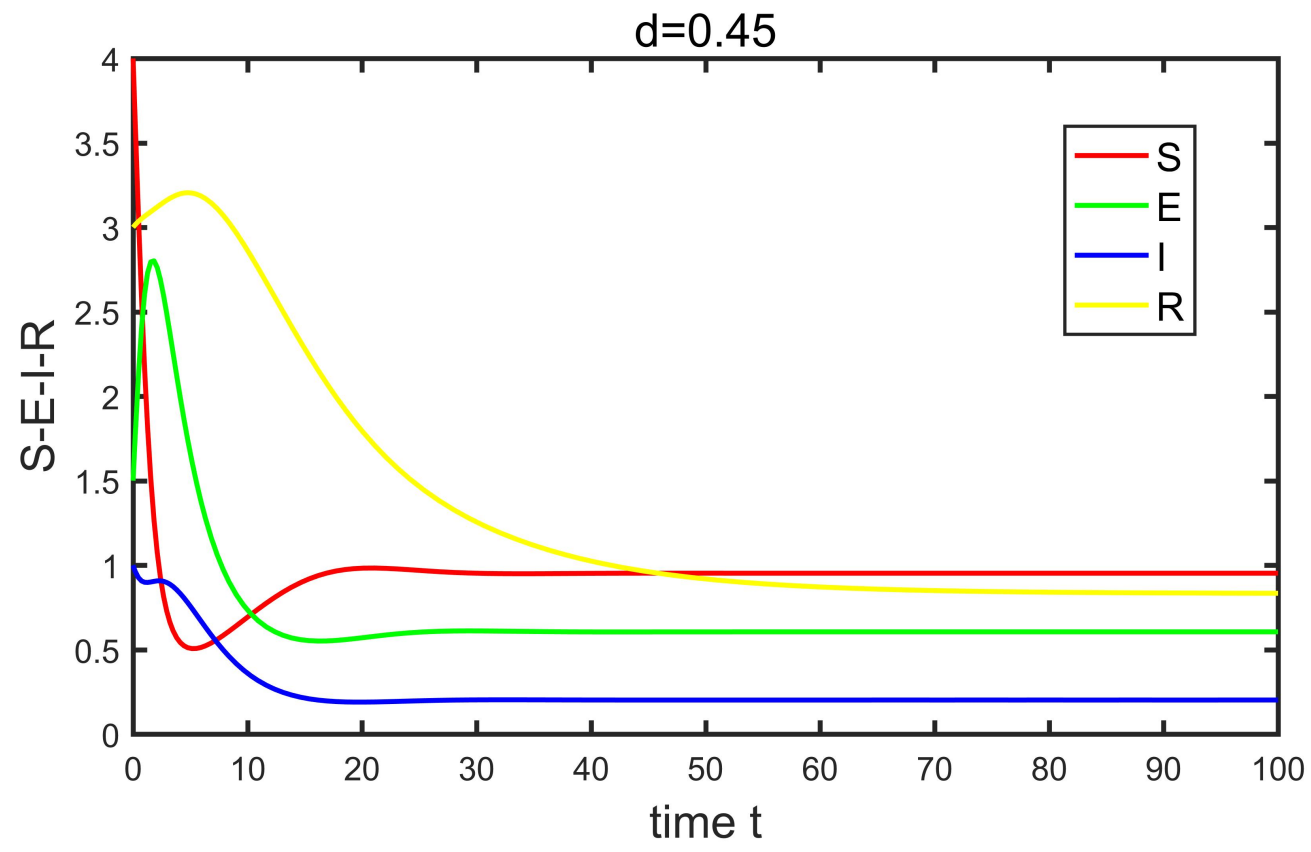

Figure 9: The endemic equilibrium point is globally asymptotically stable with $d=0.45$ which is smaller than threshold value 0.8767 .

and the extinct lag decreases as the the contacting distance increase. Figure 7(a) reveals that the minimum extinct lag of the incubation infected individuals is about 18 days, Figure 7(b) shows that the minimum extinct lag of the symptomatic infected individuals is about 28 days, since the 


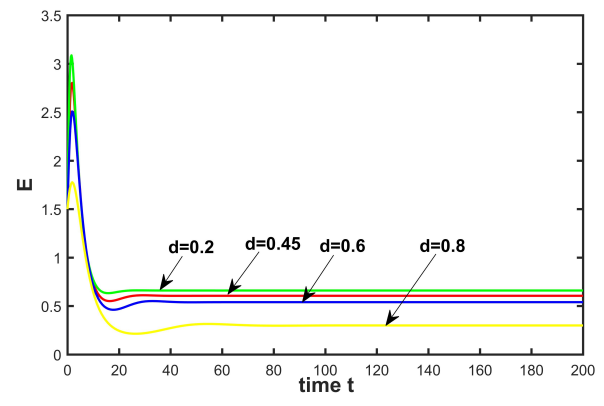

(a)

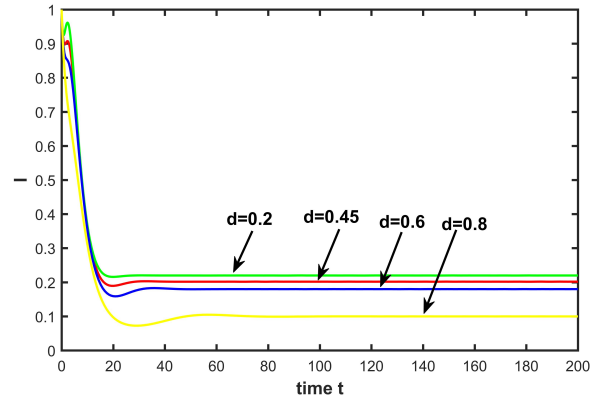

(b)

Figure 10: The time-series diagrams for $E$ and $I$ with the different contacting distance while it is smaller than the threshold value 0.8767 .

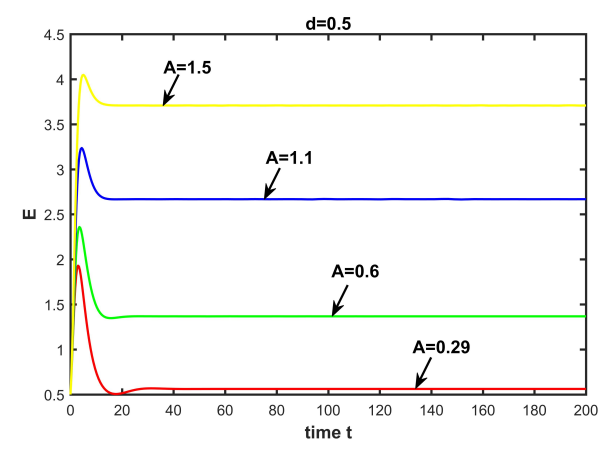

(a)

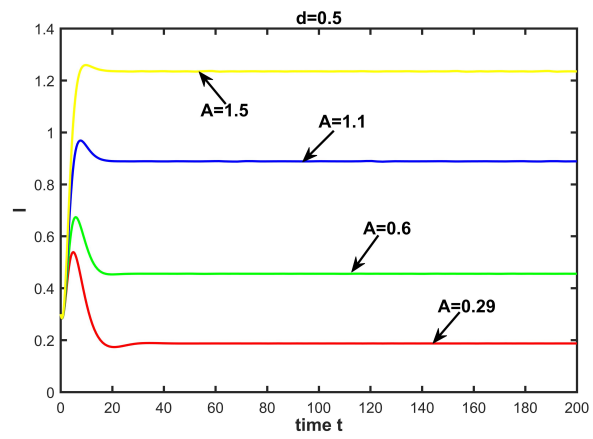

(b)

Figure 11: The time-series diagrams for $E$ and $I$ with the different immigration rate while the contacting distance is smaller than the threshold value 0.8767 .

symptomatic infected individuals must spend at least 7-14 days on being cured in hospitals and recovering in the isolated points. Figure 8 reveals that the extinct lag of the COVID-19 has the close relationship with the peoples' immigration to Whan city, and the extinct lag decreases with the peoples' immigrating number to the streets of Wuhan city decreasing. Figure 8(a) and Figure 8(b) show that the minimum extinct lag of the incubation and symptomatic infected individuals are 18 days and 24 days respectively when the peoples' immigrating number to the streets from their own home in Wuhan city is 29 percent of the total populations of Wuhan city. Moreover, the extinct lag of the COVID-19 will be become longer when some individuals immigrate to the streets of Wuhan city from the other cities $(A=1.1$ and $A=1.5$ in Figure 8(a) and Figure 8(b) respectively). Most interestingly, the outdoor-time of the active individuals has not influence on 
22 Zhihui Ma, Shufan Wang, Xuanru Lin, Xiaohua Li, Xiaotao Han, Haoyang Wang, Hua Liu

the extinction of the COVID-19, this implies that some parts of peoples could move outdoors only when they can keep just far enough contacting distance and the density of immigrating population to Wuhan city is smaller enough. Hence, it is reasonable to believe that the necessary and safe outdoor activities of the healthy individuals could not make the COVID-19 to outbreak again.

Figure 9-Figure 11 show that the COVID-19 may become endemic disease when the contacting distance smaller than the threshold value 0.8767. Figure 9 reveals that the COVID-19 will become endemic after 70 days (five times of the mean incubation period) when the contacting distance between the susceptible individuals and the symptomatic infected individuals is smaller than the threshold value 0.8767 . The endemic lag decreases as the contacting distance decreases, and hence the decrease in the contacting distance is must be encouraged. Figure 10(a) and Figure 10(b) show the minimum endemic lag of the incubation and symptomatic infected individuals are 28 days (two times of the longest incubation period). Figure 11(a) and Figure 11(b) show that the COVID-19 may become endemic disease after 38 days when the immigrating individuals to the streets from their own home in Wuhan city is 29 percent of the total populations of Wuhan city. The endemic densities of the incubation and symptomatic infected subpopulations increase and the outbreak lag of the COVID-19 decreases with the immigrating number of the susceptible individuals increasing, especially. This public events will be getting worse when some parts of peoples immigrate to Wuhan city from the other cities ( $A=1.1$ and $A=1.5$ in Figure 11(a) and Figure 11(b) respectively).

\section{Discussion}

In this paper, an SEIR-type epidemic model for the COVID-19 incorporating the contacting distance between the susceptible and asymptomatic/symptomatic infected individuals is presented. The contacting distance between the asymptomatic and symptomatic infected individuals should be explicitly incorporated into the incidence function to estimate the COVID-19. Our results show that the contacting distance between the susceptible and asymptomatic/symptomatic infected individuals plays an important role in controlling the COVID-19, and the corresponding threshold value, which can induce the COVID-19 to be extinct, is obtained by mathematical analysis based on the ordinary differential equation and the numerical simulation based on the published data. Meanwhile, the threshold value of the immigration rate is also obtained by the similar methods. The COVID-19 will be completely extinct when the contacting distance is larger than the corresponding threshold value $\tilde{d}^{*}=1-\frac{\mu\left(\mu+\mu_{1}+\gamma_{12}\right)\left(\mu+\mu_{22}+\gamma_{22}\right)}{\beta_{2} A \gamma_{12}}$ or the immigration rate is smaller than the corresponding threshold value $A^{*}=\frac{\mu\left(\mu+\mu_{1}+\gamma_{12}\right)\left(\mu+\mu_{22}+\gamma_{22}\right)}{\beta_{2}(1-d)}$ under some certain conditions. 
Researchers could estimate these threshold values based on the data of the other cities and give some positive proposals for the disease control. Furthermore, our results reveal that the average outdoortime of the asymptomatic/symptomatic infected individuals has no influence on the theoretical control for the COVID-19. Our study gives some physical and theoretical methods to control the COVID-19 and some reasonable suggestions for the policy-makers. However, our paper use the average contacting distance in the modelling processes, and does not consider the temporal and spatial effects in the contacting distance. Meanwhile, our model considers the respiratory transmission and the contacting transmission in the modelling processes only, and does not take into account the aerosol transmission. These are open issues and could be considered in the future work.

\section{Declarations}

\section{Ethics approval and consent to participate:}

The study protocol was approved by School of Mathematics and Statistics of Lanzhou University, School of Mathematics and Computer Science of Northwest Minzu University and Faculty of Science of McMaster University.

\section{Conflicts of Interest:}

The authors declare no conflict of interest.

\section{Availability of data and material:}

The data set used and/or analyzed during the current study is available from the published works.

\section{Funding}

This work was supported by Natural Science Foundation of Gansu Province (No. 20JR5RA238) and Fundamental Research Funds for the Central Universities of Northwest Minzu University (No. $31920190089)$. 
24 Zhihui Ma, Shufan Wang, Xuanru Lin, Xiaohua Li, Xiaotao Han, Haoyang Wang, Hua Liu

\section{CRediT authorship contribution statement}

Z. Ma, Conceptualization, Project administration, Writing-original draft, Writing-review and editing; S. Wang and H. Liu, Writing-review and editing, Supervision; X. Li, X. Li, Formal analysis, Software, Writing-original draft, Writing-review and editing; X. Han and H. Wang Data curation, Software.

\section{References}

[1] Bogardus, E. Measuring social distance. Journal of Applied Sociology, 1925, 9, 299-308.

[2] Chen, Y.; Liu, Q.; Guo, D. Coronaviruses: Genome structure, replication, and pathogenesis. J. Med. Virol. 2020;92:418-423.

[3] Cheng, V. C. C.; Wong, S. C.; To, K. K. W.; Ho, P. L.; Yuen, K. Y. Preparedness and proactive infection control measures against the emerging Wuhan coronavirus pneumonia in China. J. Hosp. Infect. 2020, 104(3), 254-255

[4] De Wit, E.; van Doremalen, N.; Falzarano, D.; Munster, V.J. SARS and MERS: Recent insights into emerging coronaviruses. Nat. Rev. Microbiol. 2016, 14, 523-534.

[5] Hui, D. S. C.; Zumla, A. Severe acute respiratory syndrome: Historical, epidemiologic, and clinical features. Infect. Dis. Clin. North Am. 2019, 33, 869-889.

[6] Ma, Z., Wang, S., Li, H., A generalized infectious model induced by the contacting distance, Nonlinear Anal-Real. 54 (2020) 103113.

[7] Hodgetts, D.; Stolte, O.; Radley, A.; Leggatt-Cook, C.; Groot, S.; Chamberlain, K. "Near and far": Social distancing in domiciled characterizations of homeless people. Urban Studies, 2011, 48(8), 1739-1754.

[8] Kahn, J.S.; McIntosh, K. History and recent advances in coronavirus discovery. Pediatr. Infect. Dis. J. 2005, 24, S223-227.

[9] Killerby, M. E.; Biggs, H. M.; Midgley, C. M.; Gerber, S.I.; Watson, J.T. Middle East respiratory syndrome coronavirus transmission. Emerg. Infect. Dis. 2020, 26, 191-198.

[10] Kwok, K. O.; Tang, A.; Wei, V. W. I.; Park, W. H.; Yeoh, E. K.; Riley, S. Epidemic models of contact tracing: Systematic review of transmission studies of severe acute respiratory 
syndrome and Middle East respiratory syndrome. Comput. Struct. Biotechnol. J. 2019, 17, 186-194.

[11] Li, Q.; Guan, X.; Peng, Wu P.; et al. Early Transmission Dynamics in Wuhan, China, of Novel Coronavirus-nfected Pneumonia. New. Engl. J. Med. 2020, 382(13), 1199-1207.

[12] Ma, Z.; Wang, S.; Li X. A generalized infectious model induced by the contacting distance (CTD). Nonlinear Anal. RWA. 2020, 54,103113.

[13] M. Simeone, B. H. Ian, J. R. Christian, et al. A methodology for performing global uncertainty and sensitivity analysis in systems biology, J.Theor.Biol.,254(2008), 178-196.

[14] National Health Commission of the People's Republic of China (NHCPRC). Available online: http://www.nhc.gov.cn/xcs/yqtb/202002/ac1e98495cb04d36b0d0a4e1e7fab545.shtml (Report on February $21^{t} h$ 2020).

[15] National Health Commission of the People's Republic of China (NHCPRC). Available online: http://www.nhc.gov.cn/xcs/yqtb/202001/c5da49c4c5bf4bcfb320ec2036480627.shtml (Report on January $\left.24^{t} h 2020\right)$.

[16] Rothe, C.; Schunk, M.; Sothmann, P.; et al. Transmission of 2019-nCoV infection from an asymptomatic contact in Germany. N. Engl. J. Med. 2020, 10, 1-3.

[17] Tang, B.; Wang, X.; Li, Q.; Bragazzi, N. L.; Tang, S.; Xiao, Y.; Wu, J. Estimation of the Transmission Risk of the 2019-nCoV and Its Implication for Public Health Interventions. J. Clin. Med. 2020, 9(2), 462-474.

[18] Willman, M.; Kobasa, D.; Kindrachuk, J. A Comparative analysis of factors influencing two outbreaks of middle eastern respiratory syndrome (MERS) in Saudi Arabia and South Korea. Viruses 2019, 11, E1119.

[19] Zhao, S.; Lin, Q.; Ran, J.; et al. Preliminary estimation of the basic reproduction number of novel coronavirus (2019-nCoV) in China, from 2019 to 2020: A data-driven analysis in the early phase of the outbreak. Int. J. Infect. Dis. 2020, doi:10.1016/j.ijid.2020.01.050.

[20] Annas, S.; Pratama, M. S.; Rifandi, M.; Sanusi, W.; Side, S.; Stability analysis and numerical simulation of SEIR model for pandemic COVID-19 spread in Indonesia, Chaos, Solitons and Fractals. 139 (2020) 110072. 
26 Zhihui Ma, Shufan Wang, Xuanru Lin, Xiaohua Li, Xiaotao Han, Haoyang Wang, Hua Liu

[21] Boukanjimea, B.; Caraballo, T.; Fatini, M. E.; Khalif, M. E.; Dynamics of a stochastic coronavirus (COVID-19) epidemic model with Markovian switching, Chaos, Solitons and Fractals. 141 (2020) 110361.

\section{Appendix}

Proof of Proposition 3.1.1. According to differential and integral theory, we have

$$
\frac{d}{d t}\left[S(t) \exp \left\{\int_{0}^{t} \frac{\beta(1-d(\tau)) E(\tau)}{1+\beta(1-d(\tau)) h E(\tau)} d \tau+\mu t\right\}\right]=A \exp \left\{\int_{0}^{t} \frac{\beta(1-d(\tau)) E(\tau)}{1+\beta(1-d(\tau)) h E(\tau)} d \tau+\mu t\right\}
$$

That is,

$$
S(t) \exp \left\{\int_{0}^{t} \frac{\beta(1-d(\tau)) E(\tau)}{1+\beta(1-d(\tau)) h E(\tau)} d \tau+\mu t\right\}-S(0)=\int_{0}^{t}\left[A \exp \left\{\int_{0}^{t} \frac{\beta(1-d(\tau)) E(\tau)}{1+\beta(1-d(\tau)) h E(\tau)} d \tau+\mu t\right\}\right] d t
$$

Hence,

$$
\begin{aligned}
S(t)= & \left\{S(0)+\int_{0}^{t}\left[A \exp \left\{\int_{0}^{t} \frac{\beta(1-d(\tau)) E(\tau)}{1+\beta(1-d(\tau)) h E(\tau)} d \tau+\mu t\right\}\right] d t\right\} \\
& \times \exp \left\{-\int_{0}^{t} \frac{\beta(1-d(\tau)) E(\tau)}{1+\beta(1-d(\tau)) h E(\tau)} d \tau+\mu t\right\}>0
\end{aligned}
$$

Similarly, the positivity of the other solutions $E(t), I_{A}(t), I(t)$ and $R(t)$ can be shown.

Thus, the solution $\left(S(t), E(t), I_{A}(t), I(t), R(t)\right)$ of model (2.5) with the initial conditions $(2.6)$ are positive on the finite region for all $t>0$.

Therefore, the positivity of all solutions of model (2.7) with the initial conditions (2.8) is also guaranteed since model (2.7) is a special case of model (2.5).

Proof of Proposition 3.1.2. Define $N(t)=S(t)+E(t)+I_{A}(t)+I(t)+R(t)$ and add all equations of model (2.5), it is obtained that

$$
\frac{d N}{d t}=A-\mu N-\mu_{1} E--\mu_{21} I_{A}-\mu_{22} I \leq A-\mu N
$$

By comparison theorem, it follows that

$$
0 \leq N(t) \leq \frac{A}{\mu}+N(0) e^{-d t}
$$

Thus,

$$
\limsup _{t \rightarrow+\infty} N(t) \leq \frac{A}{\mu} .
$$

Hence, all solutions $\left(S(t), E(t), I_{A}(t), I(t), R(t)\right)$ of model (2.5) with the initial conditions (2.6) are bounded on the finite region and the set $\Omega$ is a positively invariant set for model (2.5) with the initial conditions (2.6). 
Therefore, the boundness of all solutions of model (2.7) with the initial conditions (2.8) is also guaranteed since model (2.7) is a special case of model (2.5).

Proof of Proposition 3.3.1. We will prove the globally asymptotical stability of the diseasefree equilibrium point $E_{0}$. Define the following matrices:

$$
W=\left(\begin{array}{c}
S \\
E \\
I
\end{array}\right), \quad J_{E_{0}}=\left(\begin{array}{ccc}
-\mu & 0 & -\frac{\beta_{2} A(1-d)}{\mu} \\
0 & -\left(\mu+\mu_{1}+\gamma_{12}\right) & \frac{\beta_{2} A(1-d)}{\mu} \\
0 & \gamma_{12} & -\left(\mu+\mu_{22}+\gamma_{22}\right)
\end{array}\right),
$$

and

$$
J_{E_{0}}^{1}=\left(\begin{array}{c}
A-\frac{\beta_{2}(1-d) S I}{1+\beta_{2}(1-d) h_{2} I}+\frac{\beta_{2} A(1-d) E}{\mu} \\
\frac{\beta_{2}(1-d) S I}{1+\beta(1-d) h_{2}}-\frac{\beta_{2} A(1-d) E}{\mu} \\
0
\end{array}\right) .
$$

Based on the above definitions and notations, model (2.7) nearing the disease-free equilibrium point $E_{0}$ can be rewritten as:

$$
\frac{d W}{d t}=J_{E_{0}} W+J_{E_{0}}^{1} .
$$

By simple compuatation, the vector $J_{E_{0}}^{1}$ is non-positive in the set $\Omega=\{(S, I) \mid S>0, I \geq 0\}$.

Hence, combining Eq. (4.1) with the non-positivity of the vector $J_{E_{0}}^{1}$ in the set $\Omega=\{(S, I) \mid S>$ $0, I \geq 0\}$, we have

$$
\frac{d W}{d t} \leq J_{E_{0}} W
$$

According to the local stability of the disease-free equilibrium point $E_{0}$ and all characteristic roots of matrix $J_{E_{0}}$ have negative real parts.

Therefore, the disease-free equilibrium point $E_{0}$ is globally stable by the theory of the ordinary differential equation. 